\title{
Roméo et Juliette: Gounod et Shakespeare
}

\section{Francis Guinle}

\section{(2) OpenEdition \\ Journals}

Electronic version

URL: http://journals.openedition.org/shakespeare/1325

DOI: 10.4000/shakespeare.1325

ISSN: 2271-6424

\section{Publisher}

Société Française Shakespeare

\section{Printed version}

Date of publication: 1 November 1989

Number of pages: 201-214

\section{Electronic reference}

Francis Guinle, "Roméo et Juliette: Gounod et Shakespeare ", Actes des congrès de la Société française Shakespeare [Online], 7 | 1989, Online since 01 January 2007, connection on 04 May 2019. URL : http:// journals.openedition.org/shakespeare/1325 ; DOI : 10.4000/shakespeare.1325 


\title{
Société Française Shakespeare
}

\author{
Actes des Congrès \\ 1985 - 1986 - 1987
}

\section{LA FOLIE}

\section{SHAKESPEARE ET LES ARTS \\ LE TRAGIQUE}




\title{
ROMEO ET JULIETTE : GOUNOD ET SHAKES- PEARE
}

\author{
Francis GUINLE
}

Il n'est pas rare d'entendre critiquer l'opéra de Gounod comme étant peu shakespearien. Comment pourrait-il l'être ? Alors que plusieurs siècles séparent les deux œuvres, qu'elles appartiennent à deux genres très différents, dont l'un n'existait pas encore au moment où l' autre s'épanouissait, et que leur langue même diffère. On pourrait poser le problème de façon différente : que reste-t-il de Shakespeare dans l'œuvre de Gounod? Ou encore, que cherchait Gounod dans l'œuvre de Shakespeare ? Existe-t-il un véritable rapport entre les deux cuvres ?

Pour tenter de répondre à ces questions il faut replacer l'œuvre de Gounod dans son contexte, voir de quelle façon elle se démarque d'autres œuvres lyriques qui utilisent le même sujet, et comprendre la signification de ce démarquage. L'analyse de l'œuvre mise à plat, en parallèle avec la pièce de Shakespeare, révèle un souci particulier du librettiste de redécouvrir l'œuvre du dramaturge au XIXe siècle, selon les critères mêmes préconisés par Victor Hugo. Les conventions de 1'opéra trouvent-elles alors leur compte dans cette redécouverte ? Et en particulier, ce choix de Roméo et Juliette, loin d'être fortuit, n'exprime-t-il pas une tendance chez Gounod à imprimer son style dans la structure même des œuvres ? Quelques comparaisons, en particulier avec Faust et Mireille, illustrent bien ceci.

L'histoire des amants de Vérone a de nombreuses fois tenté les librettistes et les compositeurs, ceci depuis le XVIIIe siècle ; ainsi, avant l'opéra de Gounod, on en dénombre au moins 5'. Parmi eux, deux nous retiennent: Vaccaj et Bellini.

L'opéra de Vaccaj se nomme Giulietta e Romeo, et son librettiste, Romani, ne s'est pas directement inspiré de la pièce de Shakespeare, mais de chroniques italiennes du Moyen Age, relatant les querelles intestines, les rivalités des clans soutenus par telle ou telle famille de la ville. Lorsque Bellini est contacté, en catastrophe pour composer un opéra pour le carnaval de Venise de 1830, il n'a guère qu'un mois et demi pour le faire. Ayant déjà collaboré avec Felice Romani, il lui demande de remanier le livret de l'opéra de Vaccaj. Romani y apporte d'importants changements de texte, mais aussi d'intrigue, sans pour cela retoumer à Shakespeare. 
Un fait important, sur lequel nous aurons à revenir, comme dans l'opéra de Vaccaj, le rôle de Roméo est confié à une femme. A cause du succès de l'opéra de Vaccaj sans doute et de la proximité des représentations, puisque celui de Vaccaj date de 1825 , et celui de Bellini de 1830 , mais aussi probablement à cause du librettiste commun et d'une trame commune, non-shakespearienne, les deux opéras sont longtemps liés l'un à l'autre, à tel point que lors d'une reprise de l'opéra de Bellini, en 1832, la Malibran fait remplacer la dernière scène par celle de Vaccaj; la tradition persiste longtemps en Italie et passe en France pour la représentation de l'opéra en 18592. Ceci est loin d'être un cas isolé : les extrapolations d'airs, de scènes, sont fréquentes au XIXe siècle, ainsi que les modifications de tout ordre demandées aux compositeurs pour s'adapter au public (ainsi Verdi, crée des versions françaises et italiennes de certains de ses opéras, avec, en particulier des ballets pour les opéras destinés à la France).

L'opéra français suit des conventions bien définies, déjà très apparentes chez Meyerbeer ; Gounod les suit, et on trouve de nombreux échos de la musique de Meyerbeer, mais avec une structure plus ressérée, une architecture qui s'appuie sur ses conventions pour en tirer le maximum d'efficacité. dramatique.

Alors que l'opéra italien semble vouloir court-circuiter Shakespeare, la France, avec la venue des comédiens anglais, découvre, dans la langue originale l'œuvre du grand dramaturge, et ceci dès les années vingt. En France, Berlioz s'extasie sur les représentations des comédiens anglais à l'Odéon, et surtout sur Harriet Smithson qui joue les héroïnes et qu'ilépouse en 1833. Son Roméo et Juliette (1839) n'est pas un opéra, plutôt une symphonie avec voix, mais Berlioz suitla trame Shakespearienne. Ce retour à Shakespeare est bien sûr annoncé et préparé par l'œuvre dramatique de Victor Hugo, et les scandales qu'elle provoque, et par sa célèbre Préface de Cromwell de 1827. Il n'est pas surprenant de voir les pièces des deux dramaturges mis en musique par des compositeurs aussi prestigieux, à l'époque, que Rossini, Verdi, Berlioz, Ponchielli, Thomas et Gounod'.

Du reste, cen'est pas seulement Shakespeare qui fascine cette époque, mais l'Angleterre de la Renaissance, et nombreux sont les opéras, depuis le début du siècle, qui puisent leur sujet dans l'histoire de cette période, soit à travers des pièces comme le Marie Stuart de Schiller, soiten tirant de l'histoire elle-même des épisodes mettant en valeur un grand personnage au destin tragique (Anne Boleyn, Robert Devereux, Elisabeth I....).

Dès 1841, alors qu'il se trouve à Rome, à la villa Médicis, Gounod songe à un opéra ayant pour sujet les amants de Vérone4. Mais lorsqu'en 1865 il reprend ce projet, il est déjà un compositeur d'opéra confirmé, ayant composé les deux chefsd'œuvre que sont $F$ aust et Mireille. De plus, sa collaboration avec les librettistes Barbier et Carré semble particulièrement fructueuse. Ceux-ci n'hésitent pas à s'attaquer à de grandes cuvres littéraires qu'ils adaptent au goût du jour, et aux conventions du genre. Le goût du jour va justement à Shakespeare, et ils ont à leur 
disposition plusieurs traductions dont la plus récente, celle de Fançois-Victor Hugo constitue leur base de travail. L'œuvre est représentée au Thêâtre Lyrique du Châtelet en 1867 et remporte un vif succès, ce qui n'empêchera pas Gounod de la remanier à chaque reprise de son vivant : coupures, ajouts, reprises d'anciennes coupures, tout cela caractérise la version traditionnellement jouée, de 1888, pour les représentations au Palais Garnier.

On ne peut bien sûr pas séparer livret et musique lorsque l'on parle d'opéra, les deux sont intimement liés et même, comme c'est le plus souvent le cas, le livret précède l'écriture musicale, il contient déjà en grande partie le découpage musical de l'œuvre ; en effet, le librettiste travaille en fonction des conventions du genre, ou en fonction de demandes spécifiques du compositeur. Un livret donné à un musicien n'est du reste pas définitif, et des échanges constants ont lieu entre les deux artistes.

Au XIXe siècle, l'opéré français suit un découpage en actes et tableaux, dans lesquels s'enchaînent divers «numéros», divisions plus musicales que dramatiques: chœurs, dont peuvent se détacher une ou plusieurs voix pour dialoguer, solos, ensembles (duos, trios...) parfois soutenus par un chœur, dialogues sur le mode récitatif (parfois parlés, mais de plus en plus l'opéra comique se met aux passages récitatifs chantés). La continuité est assurée par l'orchestre qui ménage des transitions rythmiques ou mélodiques, indiquant ainsi l'évolution dramatique de l'œuvre, mais aussi l'évolution psychologique des personnages, évolution qui s'exprime ensuite dans les parties chantées. On voit que nous ne sommes pas loin du découpage du drame shakespearien, et on pourrait établir des correspondances quasi-générales : ainsi le chœur de l'opéra et l'orchestre peuvent assumer une des fonctions du chœur shakespearien ; les solos répondent aux tirades et monologues, les récitatifs aux échanges brefs entre deux ou plusieurs personnages, et même les scènes d'ensembles dont se détachent des voix particulières trouvent leur parallèle dans les pièces, même si la masse des voix n'articule pas vraiment un énoncé.

Certes, le librettiste, en adaptant, va opérer une restructuration des éléments. Il effectue un choix de personnages, un choix d'épisodes dramatiques particulièrement marquants ou fondamentaux, enfin un choix de textes qu'il tentera de dénaturer le moins possible, tout en les pensant musicalement.

C'est ainsi, en effet, que semblent avoir procédé les librettistes de Gounod. L'opéra débute donc, comme la pièce, par un chœur qui reprend les grandes lignes du prologue de Shakespeare, plaçant l'œuvre sous le signe inévitable de la tragédie (ceci justifie un parti pris de mise en scène tragique de bout en bout de l'opéra)s.

Le découpage de l'opéra se fait sur cinq actes qui ne suivent pas exactement les divisions traditionnelles que l'on trouve dans les éditions de la pièce de Shakespeare. Ces cinq actes portent chacun un titre qui donne une idée du contenu par rapport à la pièce. Après le prologue donc, l'œuvre s'ouvre sur le bal chez les Capulets. On voit d'emblée le souci de resserrement dramatique, et la convention du genre qui veut une grande scène spectaculaire, présentant la quasi-totalité des personnages, contrastant par sa gaité et sa vigueur avec les annonces du chœur 
d'introduction, mais laissant bien sûr présager la tragédie. Tout est mis en place à travers la scène du bal qui ici constitue l'acte tout entier. Rien ne manque, sinon un personnage, Lady Capulet. Après un chœur sur un rythme de mazurka, l'échange Tybalt/Paris vient remplacer la scène 2, où Paris demande à Capulet Juliette en mariage. Capulet accueille les invités etannonce l'apparition de Juliette, car il s'agit d'une apparition qui ravit tout le monde et entraîne un murmure d'admiration. Nous sommes à l'opéra, et Juliette se doit donc de nous donner un aperçu de son personnage vocal. Il ne s'agit pas encore d'un air à proprement parler, mais elle nous annonce une naissance à la vie, une joie de vivre toute insouciante, qu'elle confirme un peu plus loin dans un dialogue avec la nourrice, nommée Gertrude. Ici nous suivons Shakespeare pas à pas. Roméo veut partir car il a fait un rêve, et Mercutio enchaîne sur «La Ballade de la Reine Mab», dont le texte est très largement inspiré de la tirade de la pièce. Roméo aperçoit alors Juliette et en oublie Rosaline, ce que les Montaigus lui reprochent de façon narquoise. L'épisode suivant est une interpolation de la scène 3, où Lady Capulet prépare Juliette au mariage avec Paris. Juliette dialogue avec Gertrude, et au lieu de la réponse réservée de Juliette dans la pièce, nous avons un véritable hymne à la jeunesse et à l'insouciance : la très justement célèbre valse. Nous savons que Gounod l'a écrità la demande de son interprète, Mme Miolan Carvalho, femme du directeur du Thêâtre Lyrique où l'œuvre devait être représentée. On voit déjà le type de contraintes auquel les compositeurs étaient soumis. Ceci dit, Mme Miolan Carvalho devait posséder une fort belle voix que Gounod a dû prendre un grand plaisir à mettre en valeur : l'air est un des plus célèbres du répertoire français de l'époque et a été enregistré par plus de cinquante cantatrices, avec des noms aussi illustres que Nellie Melba, qui chanta le rôle en 1889, Geraldine Farra, Fanny Heldy, Lily Pons, Mado Robin, Hoan Sutherland et Maria Callas. On enchaîne alors sur le «Madrigal à deux voix», qui n'est autre que le dialogue du pèlerin, dialogue interrompu comme dans la pièce, mais ici par Tybald lui-même qui reconnait alors Roméo. Les choses vont très vite, et la révélation des identités est beaucoup plus directe que dans la pièce, puisque Juliette elle même annonce qui elle est à Roméo, et dans la foulée, Tybald apprend à Juliette le nom de son galant. Les deux clans s'affrontent un court instant et Capulet reprend les choses en main, calmant les esprits, et incitant à nouveau les invités à la danse.

Les librettistes ont tenté de faire apparaître certaines données de la pièce, nécessaires à la bonne compréhension de l'intrigue, mais en les insérant toutes dans un cadre spectaculaire propre à l'opéra. En ce sens la scène du bal se rapproche de celle de la kermesse de Faust, ou encore de la scène de fête avec la farandole de Mireille. Certes, la valse de Juliette suit une convention purement lyrique, en revanche pour la ballade de Mercutio et le duo, on peut noter les affinités qui existent entre les deux cuvres, en fait, dès le départ, le texte de Shakespeare «répond» merveilleusement aux exigences de l'opéra ; car cette ballade trouve bien son parlallèle dans Faust, avec l'air du «Veau d'or», de Mephistophélès, ou celui de la chanson du «rat» de Wagner dans la scène de la kermesse. 
Le deuxième acte s'intitule : «Le Jardin de Juliette». Dans la pièce, il s'ouvre sur un prologue, dit par le chœur qui place l'acte sous le signe de l'amour. Ce prologue devient orchestral dans l'opéra, et en quelques mesures tout le ton de la scène qui suit est donné. Roméo entre dans le jardin des Capulets ; la tirade de Mercutio est remplacée ici par un appel, et un chœur très court qui fait immédiatement place à la cavatine de Roméo : il est temps, en effet que l'on entende le ténor dans un air de bravoure. Le texte de la cavatine est un condensé habile de la tirade de Roméo :

Arise, fair sun, and kill the envious moon.

$$
\text { (II, ii, 4) }
$$

The brightness of her cheeks would shame those stars As daylight would a lamp.

$$
\text { (II, ii, 19-20)'. }
$$
Ah! lève-toi soleil ! fais pâlir les étoiles
$Q u i$ dans la nuit sans voile, brillent au firmament

Après un court monologue de Juliette apparue sur son balcon, le duo s'engage, suivant encore une fois d'assez près le texte de Shakespeare, tout au moins dans les temps forts, le nom, l'amour, le mariage ; interrompu à deux reprises par les appels de la nourrice dans la pièce, dans l'opéra ces interruptions se font plus pressantes ou dangereuses : Grégorio et les valets des Capulets sont à la recherche du Page de Roméo qu'ils ont aperçu rôdant près de la maison; ils rencontrent Gertrude, et une scène comique s'en suit ; puis la nourrice fait rentrer Juliette, qui ressort quelques instants après pour reprendre un duo à nouveau interrompu par les appels de Gertrude. Un soin particulier s'attache à conserver certains vers, certaines répliques particulièrement marquantes ou poétiques, ainsi le célèbre :

Goodnight, goodnight, parting is such sweet sorrow That I shall say goodnight till it be morrow.

$$
\text { (II, ii, 184-185). }
$$

Adieu! De cet adieu si douce est la tristesse $Q u e$ je voudrais te dire adieu jusqu'd demain.

L'acte 3 de l'opéra est l'un de ceux qui présentent le plus de bouleversements par rapport à la pièce. Son découpage en deux tableaux suit à peu près la fin de l'acte II et le début de l'acte III de la pièce, mais à l'opéra, il est pratiquement impossible d'avoir des allées et venues entre un lieu et un autre, comme c'est le cas 
dans la pièce, entre la cellule de Frère Laurent, et la place publique de Vérone (du moins cela n'était-il pas concevable dans la scénographie du XIXe siècle qui voulait un décor bien défini pour chaque acte, ou du moins chaque tableau; à l'heure actuelle on peut se permettre plus de souplesse, grâce à des moyens techniques perfectionnés, et une approche scénographique différente, et dans certains pays, en particulier en Grande Bretagne, la tendance est à une souplesse dans les décors qui permet dans la plupart des cas de limiter le nombre des entr'actes ; (ce n'est hélas pas le cas en France, où le nombre et la longueur exagérés des entr'actes, souvent injustifiés par les nécessités techniques de changement de décors ou autre, coupent complètement le spectateur du propos dramaturgique qui se perd alors dans la fonte lente des chocolats glacés).

Le premier tableau représente donc la cellule de Frère Laurent, lieu vers lequel on ne reviendra plus : ainsi donc à peine Roméo a-t-il avoué son amour pour Juliette au moine, que Juliette elle-même paraît en compagnie de Gertrude, et le Frère les marie. Nous sommes bien loin ici de l'esprit ou de la lettre du texte de Shakespeare. La musique elle-même suit, sans s'élever au-dessus, les conventions. Après un court échange entre Roméo et Frère Laurent, échange qui pourtant augure bien, l'arrivée de Juliette déclenche un trio obligé, puis une homélie de Frère Laurent, suivie du mariage proprement dit, et enfin un quatuor, car Gertrude étant rentrée, il faut bien la rentabiliser et lui donner son compte de notes à chanter. Ce type de musique à la religiosité très convenue se retrouve à la fois dans Faust et dans Mireille. C'est là peut-être le moment le plus faible de toute la partition.

Le second tableau représente une rue de Vérone, près de la maison des Capulets. Là aussi, en bien des points nous nous éloignons de la pièce. Stéphano, jeune page de Roméo, cherche son maitre, et par une chanson provocante sous les fenêtres des Capulets, attire Grégorio et les valets qui s'en prennent à luỉ, il se bat courageusement contre Grégorio, lorsqu'arrive Mercutio, qui, indigné de voir un homme s'en prendre à un enfant, se jette dans la bagarre. Tybalt arrive et le provoque à son tour ; puis Roméo entre en scène tente d'arrêter la bagarre, mais Tybalt se retourne contre lui et l'insulte ; devant l'attitude pacifiste de Roméo, Mercutio attaque Tybalt et se fait tuer par lui au moment où Roméo s'interpose. Roméo se retourne alors contre Tybalt qu'il tue. Le vieux Capulet arrive et Tybalt lui souffle ses dernières volontés. Le Prince entre et bannit Roméo. Si le cœur de la scène ne differe pas de la pièce, en revanche, les deux ajouts, au début et à la fin, sont significatifs. D'abord, le rôle de téphano, dont il faudra reparler, est ici essentiel, car le page déclenche la bagarre par sa chanson provocante et son attitude belliqueuse ; ceci reste assez proche de l'échange entre Mercutio et Benvolio dans la scène 1 de l'acte III de la pièce. Mercutio se lance dans la bagarre sans autre objet que de protéger Stéphano ; ce n'est qu'après l'arrivée de Roméo que nous retrouvons les motivations de la pièce. Arpès avoir tué Tybalt, Roméo ne s'enfuit pas. Nous sommes ici dans la convention de l'opéra : il s'agit d'un final d'acte, et tous les personnages doivent rester en scène pour apporter leur contribution dramatique et vocale. Le librettiste semble avoir éprouvé la nécessité de justifier l'attitude de 
Roméo :

Roméo- Ah! Qu'ai-je fait ? Moi ! Fuir ! Maudit par elle!

Benvolio- C'est la mort qui t'attend!

Roméo- Qu'elle vienne donc!

Un autre aspect conventionnel est la dernière volonté de Tybalt, soufflée à l'oreille de Capulet ; bien sûr, il s'agit comme nous l'apprenons par la suite, de son désir de voir Juliette mariée à Paris. mais en cet instant dramatique, nous n'en savons rien :

Tybalt- Un dernier mot! et sur votre âme... exauce-moi!

Capulet- Tu seras obéi, je t'en donne ma foi.

Ceci rappelle encore une fois Faust et la scène où Faust tue Valentin, frère de Marguerite, en duel :

Valentin - Je meurs pour elle ! J'ai sottement cherché querelle d son amant!

Scène qui se termine par les imprécations de Valentin : «Marguerite ! Sois maudite !» . Le texte, dans les deux cas nous paraît, à la lecture, d'une naiserie insupportable ; cependant, remis dans son contexte dramatique, musical et scénique, il passe sans trop de mal. La force de tels passages ne vient bien sûr pas du texte seul. Nous ne devons pas perdre de vue, en lisant un livret d'opéra, que tout est mis en musique, et à l'écoute même de l'œuvre, que tout est mis en scène (ou devrait 1'être !).

Dans un final somme toute solemnel et pathétique, la sentence d'exil est prononcée, et Roméo s'écrit, anonçant l'acte suivant :

L'exil, l'exil!

Non! je mourrai, mais je veux la revoir.

Quant aux Capulets, leur dernière exclamation montre bien que la tragédie se poursuivra jusqu'à la fin :

La paix? Non! Non! Non! Non! Jamais! 
Le quatrième acte nous transporte directement dans la chambre de Juliette, nous dispensant ainsi des allées et venues entre la chambre, la cellule de Frère Laurent, la maison des Capulets. Du reste, la présence de Roméo au moment de la sentence du Prince, rend inutile la scène 3 de l'acte III. Le duo du Rossignol et de l'alouette est beaucoup moins développé dans son texte que dans la pièce, mais ici, encore une fois, la musique prend le relais. On peut du rester parler, comme le fait Joèl-Marie Fauquet dans L'Avant-Scène, d'un thème d'amour qui réapparaît à plusieurs reprises au cours de l'opéra ${ }^{8}$. Musicalement, cette scène est un des moments les plus forts de la partition. Roméo s'enfuit, et Gertrude entre pour avertir Juliette de l'arrivée de son père. Celui-ci lui annonce que tout est prêt pour son mariage avec Paris, selon les dernières volontés de Tybalt. On laisse Frère Laurent parler seul avec Juliette. Ici, nulle question de refus de la part de Juliette, comme c'est le cas dans la pièce. La scène qui oppose Juliette à sa mère et son père, en présence de la nourrice, manque, mais elle aurait considérablement allongé l'opéra, sans vraiment apporter une contrepartie efficace dramatiquement?. Au contraire même, nous ressentons à ce point de l'opéra la nécessité de resserrer la trame dramatique : deux éléments y contribuent ; d'une part l'élimination totale du personnage de Lady Capulet de l'opéra, d'autre part la présence de Frère Laurent chez les Capulets, qui évite bien sûr un changement de décors. Le rythme s'accélère jusqu'au dénouement. Dans une courte scène, Frère Laurent s'assure de la détermination de Juliette et lui propose le philtre. Ici nous sommes en présence de deux versions différentes pour des raisons bien précises : en 1888, pour les représentations au Palais Garnier, Gounod dût, selon la tradition, écrire un ballet ; il supprime la scène et l'air de Juliette qui hésite avant de boire le poison, scène proche de la scène 3 de l'acte IV chez Shakespeare. Dramatiquement, la suppression de cette scène et l'introduction d'un ballet est désastreuse, mais le ballet représentait une contrainte absolue du Palais Garnier, et les compositeurs étrangers qui désiraient voir leurs opéras joués à Paris devaient tous se plier à cette règle ${ }^{10}$. Si nous ignorons ce ballet, un cortège nuptial et un Epithalame chanté par le chœur ${ }^{11}$, font écho aux préparatifs du mariage de la pièce, et introduisent le final, encore une fois très éloigné de Shakespeare : dans une scène très brève, on assite au début du mariage de Juliette et Paris, mariage interrompu au moment même où le comte va passer la bague au doigt de Juliette, car alors Juliette défaille et tombe inerte, tous la croyant morte.

Le cinquième et demier acte, intitulé : «Lacrypte souterraine, le tombeau», s'ouvre sur un prélude orchestral, «Le Sommeil de Juliette». En réalité, avant les représentations au Palais Garnier, un court échange entre frère Laurent et Frère Jean se plaçait juste avant, pour expliquer que Roméo n'a pu être prévenu. Après le prélude orchestral, Roméo entre, seul, dans le tombeau. Ainsi toute la scène avec Paris et les pages est supprimée de l'opéra. Le parti pris des librettistes est purement conforme à la tradition lyrique. Tous les opéras précédents s'inspiraient sinon entièrement, du moins pour cette scène finale, de sources autres que Shakespeare. Le choix est compréhensible : si Roméo meurt avant le réveil de Juliette, musicale- 
ment, la scène ne peut être satisfaisante : encore une fois un duo s'impose, qui en outre dispense de tout le reste, en particulier de la longue explication de Frère Laurent de toute l'intrigue, qui déséquilibrerait la partition. Roméo trouve donc Juliette, la croit morte et boit le poison, à ce moment Juliette se réveille, et après un court hymne au bonheur et à la vie, Roméo les rappelle à la réalité par une phrase qui change tout le contexte musical :

\section{Ah! Les parents ont tous des entrailles de pierre.}

Comme tous les autres duos de l'opéra, celui-ci est interrompu, mais de façon définitive, par la mort. Après un rappel du duo de l'acte IV, Juliette cherche le poison, mais le flacon étant vide, elle se frappe d'un coup de poignard, et les amants expirent dans les bras l'un de l'autre.

En ce qui concerne les voix, la pièce de Shakespeare est une de celles qui se prêtent le mieux aux conventions de l'opéra français. Gounod en particulier, écrit tous ces opéras avec en tête deux voix principales, celles des premiers rôles : un soprano et un ténor. Aux voix de baryton, basse et mezzo sont confiés des seconds rôles, et afin d'équilibrer la partition vocalement, lorsque, et c'est souvent le cas, le nombre de personnages féminins est insuffisant, on fait chanter un rôle masculin par une femme : c'est le cas pour Siébel dans $F$ aust, et pour Stéphano, le page de Roméo, dans Roméo et Juliette. Ici, il est vrai, le nombre de personnages masculins est particulièrement élevé, ce qui permet une bonne diversité de voix, et une palette sonore colorée dans les ensembles : trois ténors : Roméo, Tybalt, et Benvolio ; trois barytons : Mercutio, Paris et Grégorio ; trois basses : Capulet, Frère Laurent, et le Duc. Bien entendu, chacune de ces voix possède un caractère et un timbre différent, et le ténor de Roméo n'est pas le même que celui de Tybalt. Face à eux, deux sopranos : Juliette et Stéphano, et un mezzo, Gertrude qui ont bien du mal à équilibrer la partition d'autant que le volume de musique attribué à Stéphano et à Gertrude est peu important. On peut alors regretter la disparition de Lady Capulet dans l'opéra, mais on se rend vite compte de la volonté de tout centrer sur les deux protagonistes, et de structurer l'ensemble de l'opéra à partir des duos qui dominent la partition. Le reste devient accessoire, comme le prouve la faiblesse de la musique de certains personnages, même lorsqu'il s'agit d'un solo, Frère Laurent par exemple ; on peut faire exception ici pour l'air de la Reine Mab de Mercutio, et bien sûr l'air de Stéphano. Il est tout à fait remarquable que Gounod ait voulu se démarquer de la tradition en donnant le rôle de Roméo à un ténor. Jusque-là, en effet, tout au moins dans les deux opéras qui précèdent directement le sien, celui de Vaccajet celui de Bellini, le rôle était confié à une femme, dans les deux cas une voix grave, pour contraster avec le soprano de Juliette. Il y a là un problème dramatique et vocal qui, depuis l'utilisation des castrats ne cesse d'intriguer. La domination des voix hautes dans l'opéra baroque a peut-être amené l'usage de travestis au moment où les 
castrats disparaissent de la scène permettant ainsi à la voix de contralto et de mezzo de se développer, et de prendre place comme premier rôle, soit en tant que personnage masculin, soit enfin comme personnage féminin. Rappelons tout de même ici que le goût français n'a jamais été, pendant toute la période baroque et classique, pour les castrats, et certains grands rôles ont été transposés à l'époque même pour pouvoir être chantés à Paris par des ténors : c'est le cas pour le rôle d'Orphée dans l'opéra de Gluck. Dans l'opéra français du XIXe siècle, les rôles travestis existent (voir ici Stéphano), mais ils sont réservés à des personnages secondaires.

La structure de l'opéra suit des exigences particulières au genre et à la période. Chaque lieu doit être parfaitement défini par un décor spécifique, et les grands changements de décors ne peuvent donc avoir lieu qu'entre les actes ; entre les tableaux, on peut à la rigueur baisser un tabs qui permettra de mettre en place quelques éléments de décors, mais sans en changer l'ensemble. On se rend vite compte que la multiplicité des lieux dans les pièces de Shakespeare, et la facilité de se déplacer de l'un à l'autre, constitue un écueil pour les librettistes qui doivent, pour les besoins de l'opéra, donner une cohérence à chāque acte; il est également souhaitable de ne pas multiplier les entr'actes, afin de ne pas détruire la tension dramatique, et la continuité de l'action ; celle-ci n'est déjà que trop rompue par les applaudissements intempestifs des spectateurs à la suite d'un air particulièrement bien interprété, ou justement célèbre. Le parti-pris de certains metteurs-en-scène actuels est d'essayer de réduire le nombre de changement de décors complets, et donc le nombre d'entr'actes. Ils y réussissent parfois, mais rarement avec les opéras de cette époque. Le travail des librettistes a déjà consisté en un rassemblement ou rebrassage des scènes, échange ou suppression de personnages, redistribution du texte essentiel, pour que l'esprit reste, et au moins une partie de la lettre. L'analyse de Roméo et Juliette est en ce sens très révélatrice de tous ces procédés.

Une autre remarque portera sur certaines libertés prises par les librettistes, et en particulier celle qui concerne la fin de l'œuvre, car enfin, ils déclarent bien s'inspirer directement de Shakespeare. Nous avons déjà avancé des raisons pour ce choix, dans l'analyse même de la scène, il nous faut ajouter que les librettistes, comme les compositeurs devaient se plier au goût d'un public souvent très conservateur, qui parfois réussit à faire changer complètement une partie d'une œuvre : ainsi il existe des partitions de Mireille dans lesquelles Mireille ne meurt pas, mais ressucite à la voix du bien-aimé, et une partition de Mignon d'Ambroise Thomas comporte quatre dénouements, selon les versions française, italienne ou allemande ${ }^{12}$.

Plus que toute autre pièce de Shakespeare, Roméo et Juliette pouvait satisfaire Gounod et l'opéra français. On y retrouve, comme dans les opéras 
précédents du compositeur, le même mélange de gaité et de tragique, l'insouciance de la jeunesse, cette jeunesse si chère à Faust, et de l'amour naissant : musicalement et dramatiquement, Faust dans le jardin de Marguerite et Roméo dans celui de Juliette sont un seul et même héros. La pièce répond aussi aux critères attendus du romantisme : personnages fantasques, grotesques même, côtoient les protagonistes de la tragédie, et malgré le chœur, au sens grec, du début de l'opéra, le destin apparaît plus comme un hasard capricieux qui fait échouer tous les plans. Le fait de tout concentrer sur les amants fait paraître encore plus pathétique cet abandon de leur sort à deux personnages aussi ternes qu'inefficaces que sont Gertrude et Frère Laurent. L'indispensable «distance», sans laquelle l'histoire des amants ne pourrait atteindre l'universel, est fournie par le temps et l'Histoire : «Vérone vit un jour deux familles rivales», chante le chcur. Encore aujourd'hui, et malgré l'habitude que nous avons des mises en scène hardies, les transpositions choquent beaucoup à l'opéra : souvenons-nous du scandale que provoqua le $F$ aust de Jorje Lavelli au Palais Garnier en 1976.

Le Roméo et Juliette de Gounod appartient bien au XIXe siècle, mais représente peut-être ce qui se fait de mieux dans l'opéra français de cette époque, par l'utilisation dramatique de conventions déjà bien figées, mais qui ici fonctionnent comme une structure solide, capable de laisser cependant s'épanouir le drame par une fusion entre la musique et le livret (entendons par là le texte et sa distribution). La pièce de Shakespeare sous-tend la dramaturgie de l'opéra, lui donne justement la force de dépasser les simples éléments conventionnels qui, sans des effets de contrastes, de changements de tons et d'humeur, les oppositions vocales, détruiraient l'impact dramatique de l'opéra. 


\section{NOTES}

1 Dans Vincenzo Bellini (Fayard : Paris, 1981), Pierre Brunel cite les opéras de Luigi Monescalchi (1784), Zingarelli, (1796), Vaccaj (1825), Torriani (1828), Bellini (1830); voir p. 191.

2 Voir Pierre Brunel, p. 190.

3 Verdi prend deux drames de Victor Hugo pour en tirer des opéras : Ermani et Le Roi s'amuse, qui donne Rigoletto ; quant à Ponchielli, son seul ouvrage célèbre, La Gioconda, elle est tirée d'Angélo, Tyran de Padoue, mais il écrit aussi un Marion Delorme, d'après la pièce de Victor Hugo.

4 Voir Roméo et Juliette, l'Avant-scène Opéra, n. 41, mai-juin 1982, p. 24. Comme tous les «Avant-scène», celui-ci comporte une abondante documentation autour de l'opéra, mais on retiendra tout particulièrement l'excellente analyse musicale de Joël-Marie Fauquet.

5 A ce sujet, il serait intéressant de comparer deux mises en scène totalement à l'opposé l'une de l'autre : celle de Georges Lavaudant, pour l'opéra de Paris en 1982, et celle de Colin Graham pour E.N.O. à Londres, en 1981.

6 Voir L'Avant-scène Opéra, qui nous fourni tous les renseignements nécessaires sur les diverses représentations de l'œuvre depuis sa création, et tous les enregistrements effectués avant 1982. Il n'y manque que le demier en date, avec Alfredo Kraus dans le rôle de Roméo et Catherine Maltifano dans celui de Juliette.

On trouvera une discographie commentée dans Opera on Record 2, ed. Alan Blith, (Hutchinson, Londres : 1983), pp. 195-207, toujours sans le dernier enregistrement en date ; ainsi que dans Opéra Quaterly, vol. 3, n. 2 (1985), pp. 100-101.

7 Les références à la pièce de Shakespeare sont de l'édition pour le Club Français du Livre, qui utilise le texte anglais du New Shakespeare, ed. Sir Arthur Quiller-Couch et John Dover Wilson, C.U.P. ; Paris, 1955.

8 Pour l'analyse des thèmes, voir L'Avant-Scène Opéra..

9 Si l'on suit le texte de Shakespeare pas à pas, la scène aurait nécessité un échange entre Lady Capulet et Juliette, probablement un solo de Lady Capulet, un solo de Juliette, un échange entre Lady Capulet, Capulet et Juliette, un solo de Capulet, un échange Juliette et Gertrude, et un solo de Gertrude.

10 Pensons aux ouvrages de Verdi, tel que Moïse, Don Carlos, Les Vêpres siciliennes.

11 Le Cortège nuptial et l'Epithalame sont souvent coupés en représentation.

12 Le livret de Mignon est également de Barbier et Carré. L'opéra fut donné pour la première fois à l'Opéra comique, en 1866. Ambroise Thomas est le compositeur d'Hamlet. 